\title{
Comparison of delta-shaped anastomosis and Billroth I reconstruction after laparoscopic distal gastrectomy for gastric cancer
}

\author{
$\mathrm{Ji} \mathrm{Li}^{1{ }^{1 *}}$, Ying-Gang Ge ${ }^{1 \#}$, Yun-Fei Yang ${ }^{2}$, Jun Zhang ${ }^{1}$ \\ ${ }^{1}$ Department of Gastrointestinal Surgery, the First Affiliated Hospital of Chongqing Medical University, Chongqing, China; ${ }^{2}$ Department of \\ Geriatrics, the First Affiliated Hospital of Chongqing Medical University, Chongqing, China \\ Contributions: (I) Conception and design: J Li; (II) Administrative support: J Zhang; (III) Provision of study materials or patients: YG Ge; (IV) \\ Collection and assembly of data: J Li; (V) Data analysis and interpretation: YF Yang; (VI) Manuscript writing: All authors; (VII) Final approval of \\ manuscript: All authors. \\ \#These authors contributed equally to this work. \\ Correspondence to: Jun Zhang. Department of gastrointestinal Surgery, the First Affiliated Hospital of Chongqing Medical University, Chongqing, \\ China. Email: Zjun2323@sina.cn.
}

\begin{abstract}
Background: This study intends to compare the short-term effects and long-term survival of gastric cancer patients who underwent delta-shaped anastomosis (DA) and Billroth I reconstructions after laparoscopic distal gastrectomy.

Methods: We retrospectively collected data from 257 patients with gastric cancer who underwent laparoscopic distal gastrectomy between January 2013 and December 2017. Patients were classified into 2 groups according to the reconstruction method used: the DA group ( $\mathrm{n}=91)$ and the Billroth I group ( $\mathrm{n}=166)$. The clinical data, short-term efficacy, and long-term results were compared between the 2 groups.

Results: The operation time $(\mathrm{P}<0.001)$ and the post-operative length of hospital stay $(\mathrm{P}<0.001)$ were shorter in the DA group than in the Billroth I group. The time to the first oral intake of a soft diet after surgery was earlier in the DA group than in the Billroth I group $(\mathrm{P}=0.014)$. Kaplan-Meier $(\log$-rank test) analysis showed no significant difference in the 5 -year survival rates between the 2 groups for patients at the same pathological stage. Multivariate analysis showed that abnormal carcinoembryonic antigen (CEA) $(\mathrm{P}=0.006)$, chemotherapy $(\mathrm{P}<0.001)$, $\mathrm{T}$ stage $(\mathrm{P}<0.001)$, and $\mathrm{N}$ stage $(\mathrm{P}<0.001)$ were independent prognostic factors for survival.

Conclusions: DA and Billroth I are feasible and safe reconstruction methods of the digestive tract after gastric cancer. DA is the recommended reconstruction method for laparoscopic distal gastrectomy.
\end{abstract}

Keywords: Gastric cancer; laparoscopic distal gastrectomy; delta-shaped anastomosis; Billroth I; outcome assessment

Submitted Jan 21, 2021. Accepted for publication Apr 04, 2021.

doi: 10.21037/jgo-21-109

View this article at: http://dx.doi.org/10.21037/jgo-21-109

\section{Introduction}

Gastric cancer remains the $6^{\text {th }}$ most prevalent form of cancer and the $3^{\text {rd }}$ leading cause of cancer-related death, with just under 800,000 new cases in 2017 alone $(1,2)$. In order to prolong the survival of gastric cancer patients, it is vital that the disease is detected early and is rapidly treated via surgical intervention $(3,4)$. Although open surgery can effectively remove the lesion, it can also result in significant trauma and a large amount of intraoperative blood loss, which is not conducive to postoperative recovery (5). At present, laparoscopic gastrectomy is a popular method. Clinical results indicate that laparoscopic 
gastrectomy has the advantages of small incision, limited tissue damage, and less intraoperative blood loss, and can clearly observe the complex structure around the stomach $(6,7)$. For laparoscopic gastrectomy, the reconstruction of the digestive tract has been of widespread concern. In order to eliminate the tiny laparoscopic scars caused by laparoscopic assisted distal gastrectomy (LADG) with extracorporeal anastomosis, intracorporeal anastomosis is currently adopted, which leads to totally laparoscopic distal gastrectomy (TLDG). In 2002, Kanaya et al. designed a totally laparoscopic reconstruction of Billroth I, called the delta-shaped anastomosis (DA) (8). It has become a common method of digestive tract reconstruction following laparoscopic distal gastrectomy. Short-term outcomes of DA have been assessed previously, however, one study lacked long-term survival results(9) and another study indicated that there were no significant differences between the short-term efficacy and long-term survival of DA and Billroth I (10), with no further confirmation. In our study, a comparison of the short-term effects and long-term survival of gastric cancer patients using DA and Billroth I after laparoscopic distal gastrectomy was performed. We present the following article in accordance with the STROBE reporting checklist (available at http://dx.doi.org/10.21037/ jgo-21-109).

\section{Methods}

\section{Patients}

Patients with gastric cancer undergoing laparoscopic distal gastrectomy from January 2013 to December 2017 were included in the study. The principle of surgery was primarily defined according to the treatment guidelines for gastric cancer in Japan $(11,12)$. All the operations were performed with D2 lymphadenectomy, and a negative gastric margin after surgery was ensured to achieve adequate tumor resection $(13,14)$. The principle of chemotherapy was based on the National Comprehensive Cancer Network (NCCN) guidelines $(15,16)$. None of the patients received radiotherapy after surgery. Patient information was obtained from the Electronic Case System of the First Affiliated Hospital of Chongqing Medical University, and phone calls or outpatient visits were used for the follow-up of all patients through to December 31, 2018.

Inclusion criteria: (I) pathological biopsy confirmed gastric adenocarcinoma; (II) preoperative chest X-ray, CT, and other auxiliary examinations did not discover cancer metastasis; (III) surgical methods: laparoscopic distal gastrectomy with D2 lymphadenectomy, while digestive tract reconstruction was with DA or Billroth I.

Exclusion criteria: (I) patients with TNM [Union for International Cancer Control [UICC]/American Joint Committee on Cancer (AJCC)] stage IV; (II) patients who refused follow-up after surgery; (III) patients who died due to tumor-independent diseases, such as trauma, car accident, or cardiovascular and cerebrovascular events.

\section{Surgical procedure}

After the patient was anesthetized, a $1 \mathrm{~cm}$ arc incision was made in the lower edge of the navel to establish a $\mathrm{CO} 2$ artificial pneumoperitoneum. Surgery was performed with a five-hole method. DA in laparoscopic distal gastrectomy was performed with just a laparoscopic linear stapler ${ }^{8}$. In the Billroth I group, after fully mobilizing the stomach and lymph nodes, a small $5 \mathrm{~cm}$ incision was made $3 \mathrm{~cm}$ below the xiphoid process. The incision was protected with a wound protector. After delivery from the pylorus, a purse suture was placed in the duodenal balloon and connected to the mushroom head of the circular stapler. The stomach was divided by the linear stapler and combined with the circular stapler to the duodenal stump.

This retrospective study included 257 patients. Among them, there were 166 cases of Billroth I and 91 cases of DA. The study was approved by the First Affiliated Hospital of Chongqing Medical University Ethics Committee (No.2018-004). All procedures performed in this study involving human participants were in accordance with the Declaration of Helsinki (as revised in 2013). Individual consent for this retrospective analysis was waived.

\section{Materials and follow-up}

Herein, the short-term effects and long-term survival of DA and Billroth I following laparoscopic distal gastrectomy for gastric cancer were compared. Patient information was collected from the case system, including age, gender, laboratory findings, tumor size, body mass index (BMI), number of lymph node metastases, human epidermal growth factor receptor 2 (HER2) expression, operative time, blood loss, postoperative complications, first anal exhaust time, time of first intake of fluid diet, postoperative hospital stay, and survival outcome. Follow-up visits were mainly conducted through telephone interviews or outpatient visits. 


\section{Statistical analyses}

SPSS v23.0 (SPSS Inc., IL, USA) was used for all statistical analyses. For quantitative variables, the normal distribution was verified. If the distribution was normal, continuous variables were compared using t-tests and were expressed as mean $\pm \mathrm{SD}$. If not, variables were tested by the Wilcoxon nonparametric test. For categorical data, a chi-square test or Fisher's exact test was utilized. The Kaplan-Meier approach and $\log$ rank tests were used for survival analyses. Cox proportional hazard regression model was used to analyze all data for univariate and multivariate analyses to verify independent survival prognostic factors. $\mathrm{P}<0.05$ was the significance threshold.

\section{Results}

\section{Clinical information}

The clinical parameters of the DA group were compared with the Billroth I group. When comparing these groups, no significant differences were detected with respect to age, gender, BMI, disease course, tumor invasion, tumor differentiation, tumor diameter, or tumor histological type. However, the preoperative platelet counts were significantly different between the DA and Billroth I groups $(\mathrm{P}=0.026)$ (Table 1). The intraoperative and postoperative parameters of the DA group were also compared with those of the Billroth I group (Table 2). The results indicated that bleeding volume, reoperation, postoperative complication rates, and postoperative recurrence rates were comparable between the DA and Billroth I groups. The median operative time (190 minutes) was reduced in the DA group relative to the Billroth I group (211 minutes) $(\mathrm{P}<0.001)$. The postoperative hospitalization duration was shorter in the DA group (6 days) relative to the Billroth I group (8 days) $(\mathrm{P}<0.001)$, and the median time to first soft diet oral intake in the DA group (5 days) was earlier than the Billroth I group (7 days) $(\mathrm{P}=0.014)$.

\section{Survival results}

A staged subgroup analysis of 5-year survival rates showed that for patients with stage I gastric cancer, the rates for patients in the DA and Billroth I groups were $93.77 \%$ and 94.44\%, respectively ( $\mathrm{P}=0.912$; Figure 1$)$. In stage II gastric cancer patients, the 5 -year survival rates in the DA and Billroth I groups were $81.07 \%$ and $73.22 \%$, respectively $(\mathrm{P}=0.477$; Figure 2). Furthermore, in stage III gastric cancer patients, the 5 -year survival rates in the DA and Billroth I groups were $63.97 \%$ and $55.38 \%$, respectively $(\mathrm{P}=0.243$; Figure 3). Differences in survival between these 2 groups were not significant among individuals of the same pathological stage.

A univariate analysis was initially performed in order to explore the prognostic relevance of clinical and perioperative variables. This analysis revealed that albumin levels $(\mathrm{P}=0.002)$, carcinoembryonic antigen (CEA) abnormality $(\mathrm{P}=0.007)$, tumor size $(\mathrm{P}=0.024)$, chemotherapy $(\mathrm{P}=0.009)$, $\mathrm{T}$ stage $(\mathrm{P}<0.001)$, and $\mathrm{N}$ stage $(\mathrm{P}<0.001)$ were all associated with patient survival outcomes. These parameters were then incorporated into a subsequent multivariate analysis, which indicated that CEA abnormality $(\mathrm{P}=0.006)$, chemotherapy $(\mathrm{P}<0.001)$, $\mathrm{T}$ stage $(\mathrm{P}<0.001)$, and $\mathrm{N}$ stage $(\mathrm{P}<0.001)$ were all independent predictors of patient survival outcomes (Table 3).

\section{Discussion}

Common gastrointestinal reconstruction methods after laparoscopic distal gastrectomy include DA and Billroth I. The ideal method of anastomosis not only achieves the purpose of treating tumors and reconstructing the digestive tract, but also ensures good gastrointestinal function and reduces the rates of adverse outcomes or death following surgery. It is essential that significant attention be paid to the postoperative quality of life and prognosis of these patients $(17,18)$. In clinical practice, Billroth I is commonly used by surgeons in the Asia Pacific region (19). Billroth I reconstruction was required to complete the anastomosis of the residual stomach and duodenum by laparoscopic assistance, while DA was totally performed under laparoscopy. TLDG has the advantages of small incision and minimal tissue damage $(6,7)$. Therefore, the operation time of DA is shorter, the postoperative wound recovery is faster, and the postoperative hospitalization duration is shorter. The median operative time (190 minutes) was shorter in the DA group than in the Billroth I group (211 minutes) $(\mathrm{P}<0.001)$. The post-operative length of hospital stay in the DA group (6 days) was reduced compared with the Billroth I group ( 8 days $)(\mathrm{P}<0.001)$. The median time to first soft diet oral intake (5 days) in the DA group was earlier than in the Billroth I group (7 days) $(\mathrm{P}=0.014)$. These results are consistent with the results obtained in the previous literature (9).

Long-term survival of different anastomotic methods have been of widespread concern. Our results indicated 
Table 1 Comparison of clinical characteristics

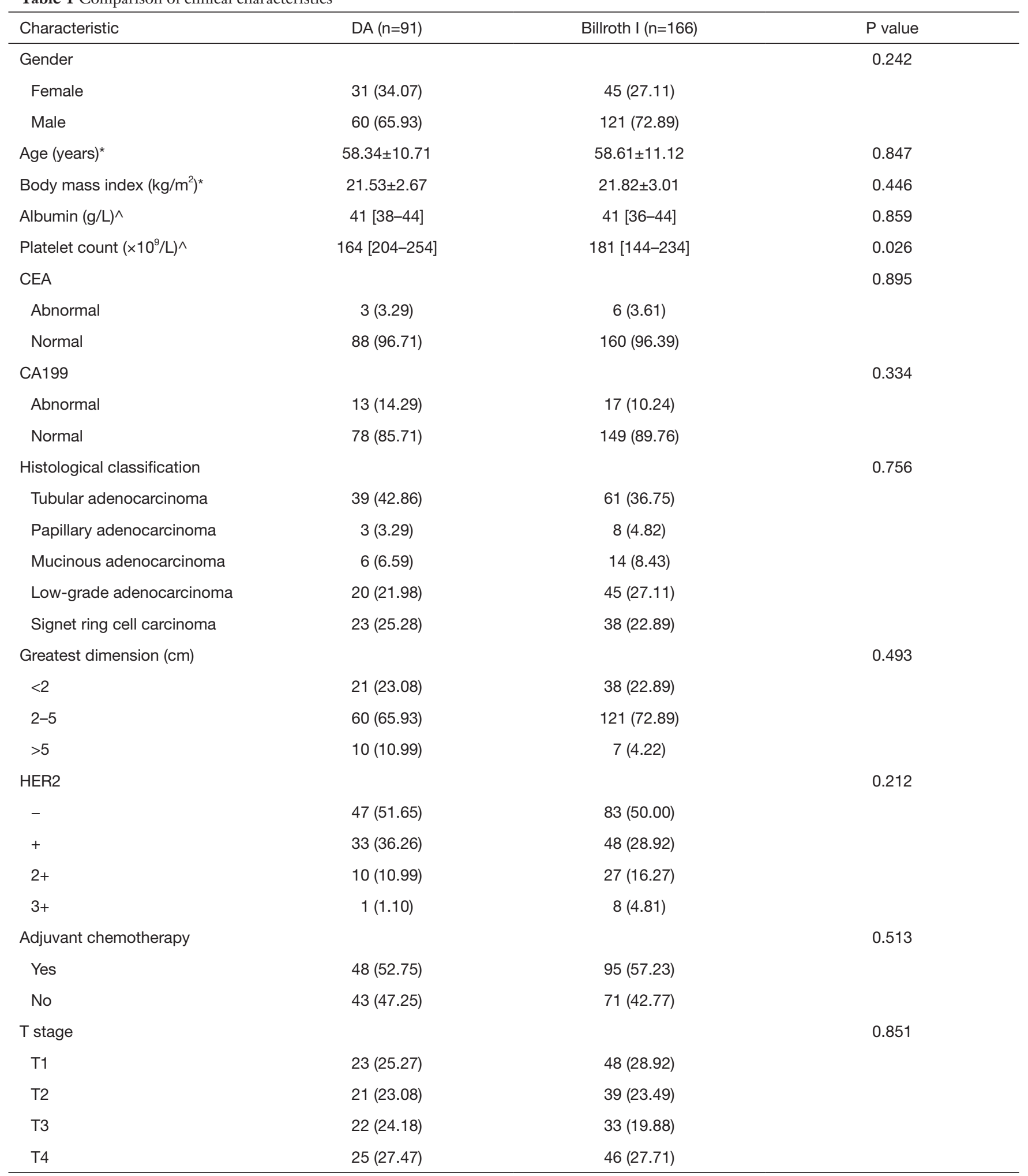

Table 1 (continued) 
Table 1 (continued)

\begin{tabular}{|c|c|c|c|}
\hline Characteristic & $\mathrm{DA}(\mathrm{n}=91)$ & Billroth I $(n=166)$ & $P$ value \\
\hline $\mathrm{N}$ stage & & & 0.433 \\
\hline NO & $57(62.63)$ & 88 (53.02) & \\
\hline $\mathrm{N} 1$ & $12(13.19)$ & 30 (18.07) & \\
\hline N2 & 10 (10.99) & $26(15.66)$ & \\
\hline Stage I & $37(40.66)$ & 66 (39.76) & \\
\hline Stage II & $21(23.08)$ & 39 (23.49) & \\
\hline Stage III & $33(36.26)$ & $61(36.75)$ & \\
\hline
\end{tabular}

Continuous variables were analyzed using the two-sample $t$-test or the Wilcoxon rank-sum test. Categorical variables were analyzed using the chi-square test. Unless indicated otherwise, data are the number of patients, with percentages in parentheses. * data are means \pm standard deviations. $\wedge$, data are medians, with interquartile ranges in parentheses. DA, delta-shaped anastomosis.

Table 2 Patients' intraoperative and postoperative parameters

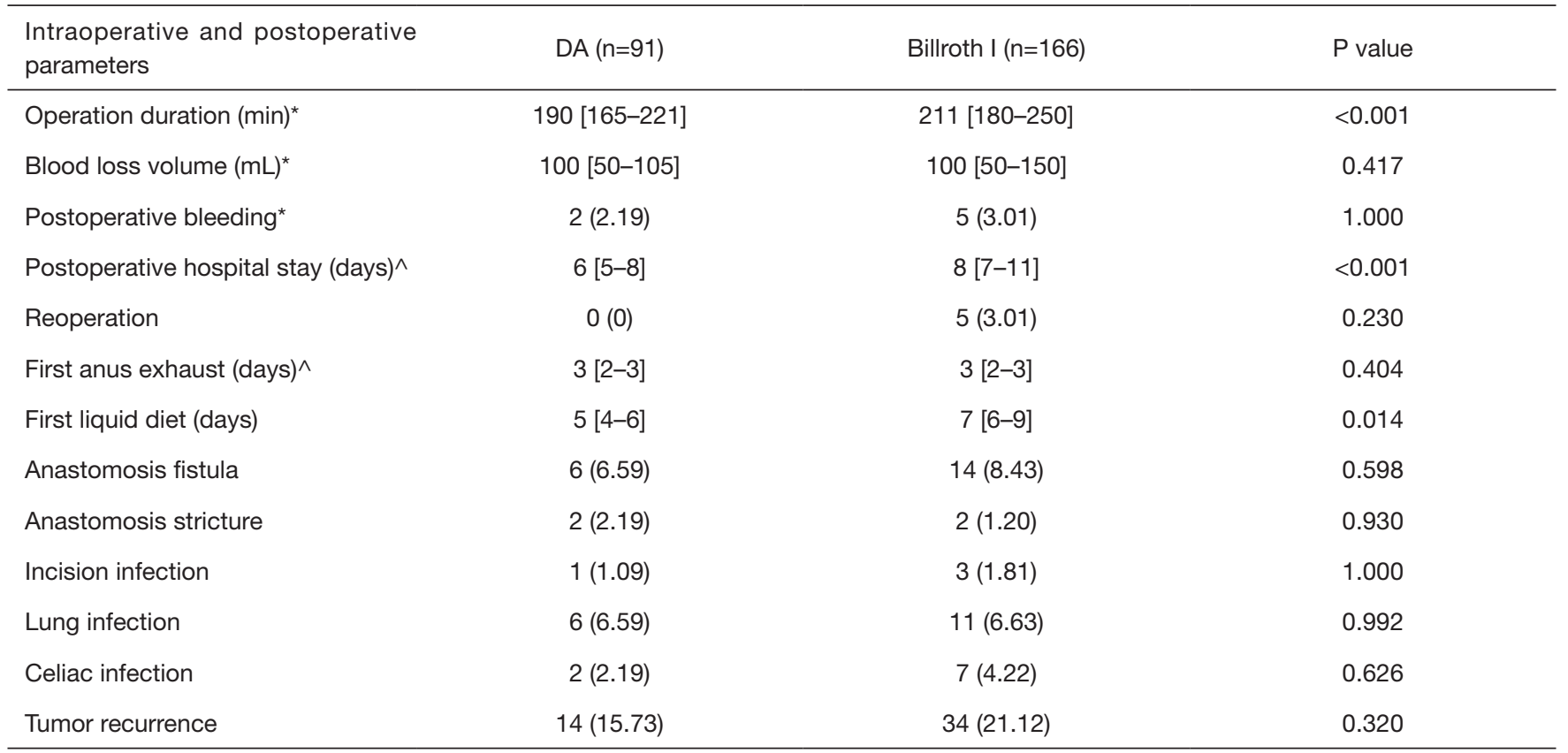

Continuous variables were analyzed using the two-sample $t$-test or the Wilcoxon rank-sum test. Categorical variables were analyzed using the chi-square test. Unless indicated otherwise, data are the number of patients, with percentages in parentheses. *, data are means \pm standard deviations. $\wedge$, data are medians, with interquartile ranges in parentheses. DA, delta-shaped anastomosis.

that 5-year survival rates were comparable between the DA and Billroth I groups. A staged subgroup analysis of 5 -year survival rates showed that for patients with stage I gastric cancer, the rates for patients in the DA and Billroth I groups were $93.77 \%$ and $94.44 \%$, respectively $(\mathrm{P}=0.912$; Figure 1). In stage II gastric cancer patients, the 5-year survival rates for patients in the DA and Billroth I groups were $81.07 \%$ and $73.22 \%$, respectively ( $\mathrm{P}=0.477$; Figure 2$)$. In stage III gastric cancer patients, the rates for patients in the DA and Billroth I groups were 63.97\% and 55.38\%, and $32.21 \%$, respectively $(\mathrm{P}=0.243$; Figure 3$)$. Differences in survival between these 2 groups were not significant among 


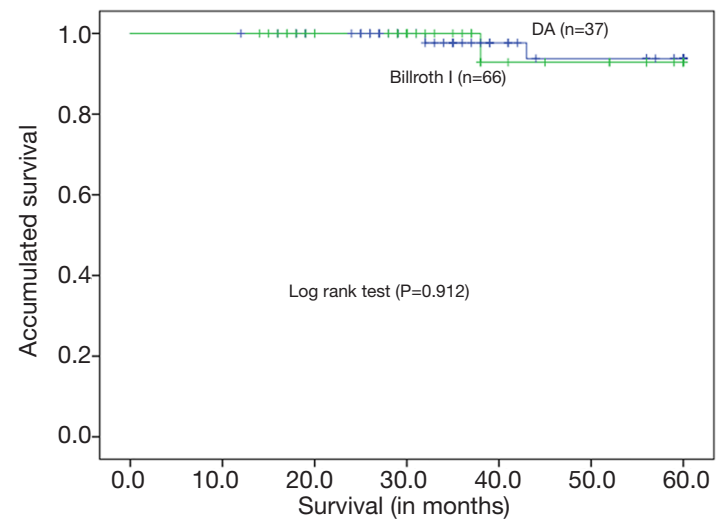

Figure 1 In the staged stratified subgroup analysis of stage I gastric cancer patients, the 5-year survival rates of the DA group and the Billroth I group were $93.77 \%$ and $94.44 \%$, respectively $(\mathrm{P}=0.912)$. DA, delta-shaped anastomosis.

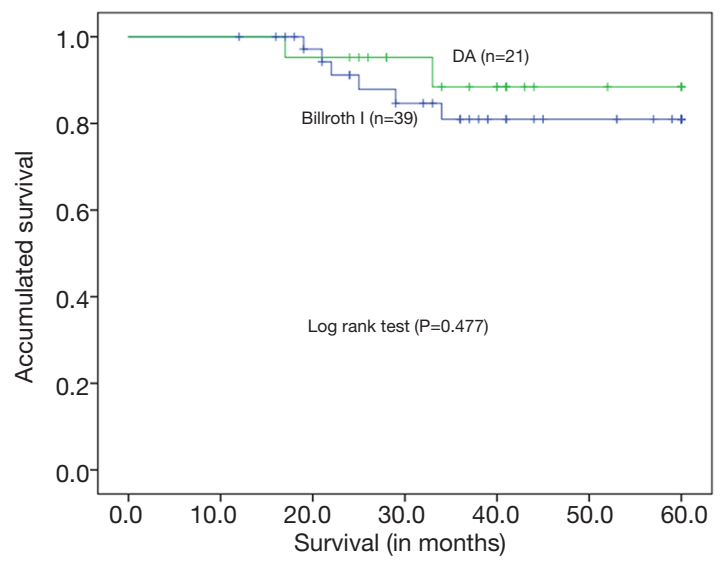

Figure 2 In the staged stratified subgroup analysis of stage II gastric cancer patients, the 5-year survival rates of the DA group and the Billroth I group were $81.07 \%$ and $73.22 \%$, respectively $(\mathrm{P}=0.477)$. DA, delta-shaped anastomosis.

individuals of the same pathological stage.

The prognostic relevance of specific clinicopathological variables was assessed using univariate and multivariate analyses, which revealed that for both groups, CEA abnormality $(\mathrm{P}=0.006)$, chemotherapy $(\mathrm{P}<0.001)$, $\mathrm{T}$ stage $(\mathrm{P}<0.001)$, and $\mathrm{N}$ stage $(\mathrm{P}<0.001)$ were independent prognostic factors for survival (Table 3). CEA is one of the most commonly used serum tumor markers in gastric cancer. Previous studies have confirmed the value of CEA for predicting the prognosis of gastric cancer patients $(20,21)$. Similarly, T stage and N stage grounding represent

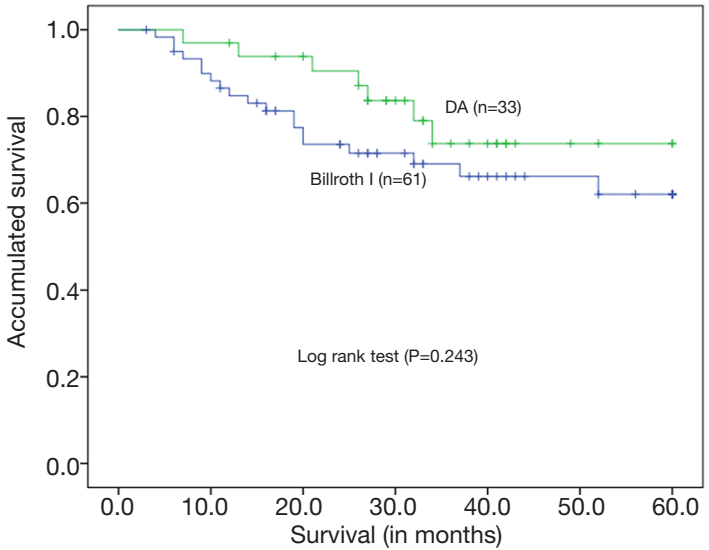

Figure 3 In the staged stratified subgroup analysis of stage III gastric cancer patients, the 5-year survival rates of the DA group and the Billroth I group were $63.97 \%$ and $55.38 \%$, respectively ( $\mathrm{P}=0.243)$. DA, delta-shaped anastomosis.

the extent of tumor progression and TNM staging, and postoperative chemotherapy affects postoperative tumor recurrence and progression. Therefore, these factors may affect survival outcomes.

There are multiple limitations to the present study. Firstly, this was a retrospective analysis and it is therefore susceptible to indication and selection biases. The reconstruction of laparoscopic distal gastrectomy for gastric cancer is determined by the surgeon. They usually choose Billroth I as the method for gastrointestinal reconstruction, resulting in differences in the total sample size of each group. Secondly, when we calculated postoperative survival, we did not detail the long-term complication rates of patients, and the patients' long-term nutritional status, gastrointestinal function, and quality of life were not compared between treatment groups. However, multivariate and phased subgroup analyses were conducted, and these results were nonetheless convincing. Further prospective randomized controlled trials will be essential in order to better explore the optimal gastrointestinal reconstruction approach for LADG.

In summary, our results indicate that it is possible to safely conduct DA and Billroth I reconstruction following laparoscopic distal gastrectomy while achieving satisfactory complication rates. Among patients with diseases of the same pathological stage, no significant differences in 5 -year survival were detected. DA offers clear advantages as a reconstruction approach, including the fact that it is a laparoscopic approach that results in minimal scarring 
Table 3 Prognostic factors for survival

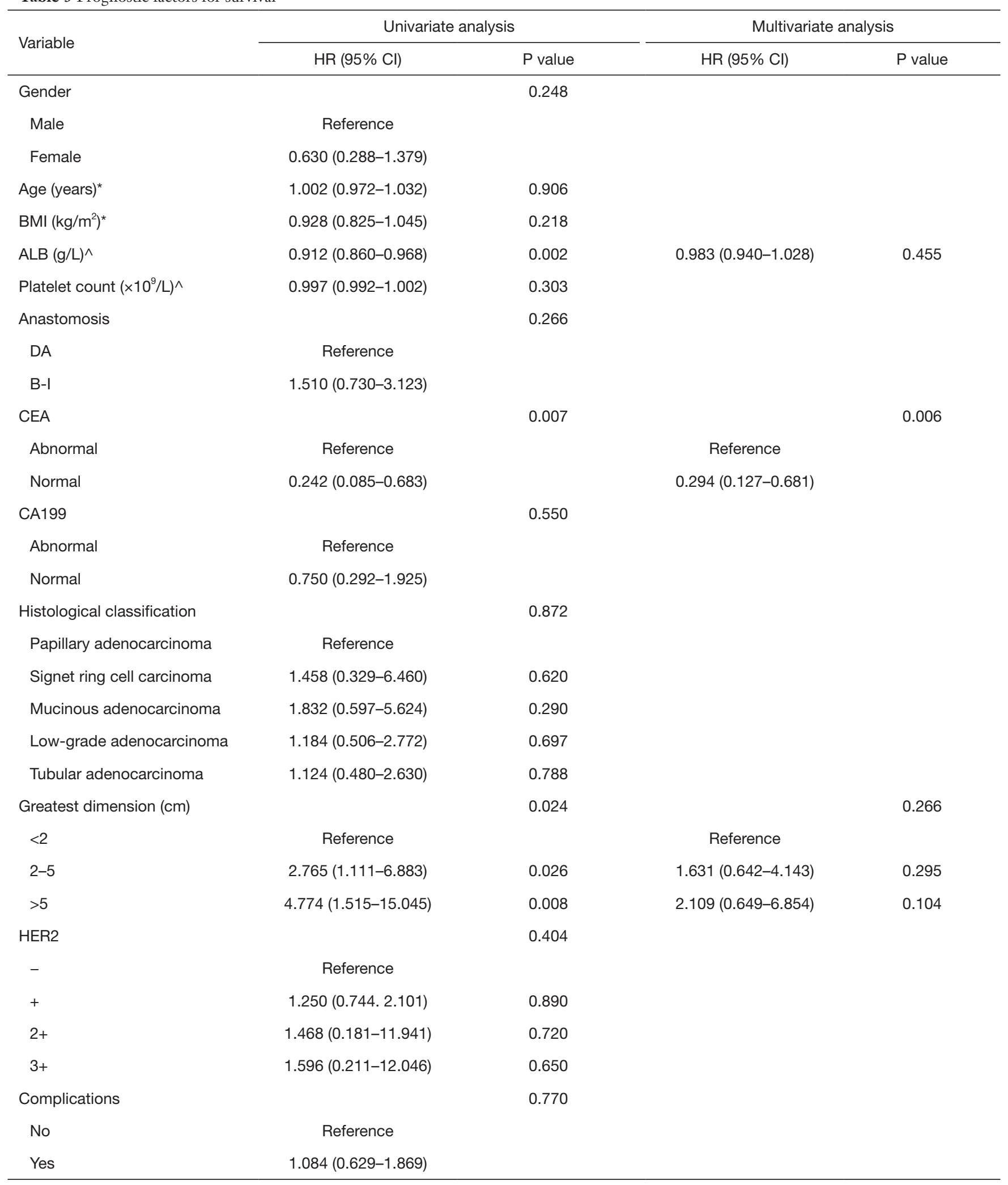

Table 3 (continued) 
Table 3 (continued)

\begin{tabular}{|c|c|c|c|c|}
\hline Variable & \multicolumn{2}{|c|}{ Univariate analysis } & \multicolumn{2}{|c|}{ Multivariate analysis } \\
\hline Adjuvant chemotherapy & & 0.009 & & $<0.001$ \\
\hline Yes & Reference & & Reference & \\
\hline No & $0.414(0.215-0.799)$ & & $2.145(1.630-2.821)$ & \\
\hline T1 & Reference & & Reference & \\
\hline $\mathrm{T} 2$ & $2.470(0.844-7.227)$ & 0.099 & $2.834(0.947-8.488)$ & 0.063 \\
\hline T3 & $6.937(2.655-18.126)$ & $<0.001$ & $5.941(2.183-16.167)$ & $<0.001$ \\
\hline $\mathrm{T} 4$ & 7.669 (3.004-19.733) & $<0.001$ & 7.418 (2.674-20.581) & $<0.001$ \\
\hline $\mathrm{N} 1$ & $2.102(0.970-4.554)$ & 0.060 & $2.320(1.041-5.166)$ & 0.039 \\
\hline N2 & 4.760 (2.515-9.008) & $<0.001$ & $4.086(2.074-8.051)$ & $<0.001$ \\
\hline N3 & $7.476(4.067-13.744)$ & $<0.001$ & $4.472(2.340-8.548)$ & $<0.001$ \\
\hline
\end{tabular}

Numbers in parentheses are $95 \%$ Cls. HR, hazard ratio. Cl, Confidence interval. DA, delta-shaped anastomosis.

while also reducing hospitalization duration and operative duration. Furthermore, DA patients exhibited more rapid postoperative dietary recovery than Billroth I patients.

\section{Acknowledgments}

The authors thank Dr. Zeng and Dr. Wu for reviewing an early draft of this article and providing valuable comments. Funding: None.

\section{Footnote}

Reporting Checklist: The authors have completed the STROBE reporting checklist. Available at http://dx.doi. org/10.21037/jgo-21-109

Data Sharing Statement: Available at http://dx.doi. org/10.21037/jgo-21-109

Conflicts of Interest: All authors have completed the ICMJE uniform disclosure form (available at http://dx.doi. org/10.21037/jgo-21-109). The authors have no conflicts of interest to declare.

Ethical Statement: The authors are accountable for all aspects of the work in ensuring that questions related to the accuracy or integrity of any part of the work are appropriately investigated and resolved. The study was approved by the First Affiliated Hospital of Chongqing Medical University Ethics Committee (No. 2018-004). All procedures performed in this study involving human participants were in accordance with the Declaration of Helsinki (as revised in 2013). Individual consent for this retrospective analysis was waived.

Open Access Statement: This is an Open Access article distributed in accordance with the Creative Commons Attribution-NonCommercial-NoDerivs 4.0 International License (CC BY-NC-ND 4.0), which permits the noncommercial replication and distribution of the article with the strict proviso that no changes or edits are made and the original work is properly cited (including links to both the formal publication through the relevant DOI and the license). See: https://creativecommons.org/licenses/by-nc-nd/4.0/.

\section{References}

1. Bray F, Ferlay J, Soerjomataram I, et al. Global cancer statistics 2018: GLOBOCAN estimates of incidence and mortality worldwide for 36 cancers in 185 countries. CA 
Cancer J Clin 2018;68:394-424.

2. Ferro A, Peleteiro B, Malvezzi M, et al. Worldwide trends in gastric cancer mortality (1980-2011), with predictions to 2015 , and incidence by subtype. Eur J Cancer 2014;50:1330-44.

3. Kim HH, Han SU, Kim MC, et al. Long-term results of laparoscopic gastrectomy for gastric cancer: a large-scale case-control and case-matched Korean multicenter study. J Clin Oncol 2014;32:627-33.

4. Sakakura C, Takemura M, Hagiwara A, et al. Overexpression of dopa decarboxylase in peritoneal dissemination of gastric cancer and its potential as a novel marker for the detection of peritoneal micrometastases with real-time RT-PCR. Br J Cancer 2004;90:665-71.

5. Caruso S, Patriti A, Roviello F, et al. Robot-assisted laparoscopic vs open gastrectomy for gastric cancer: Systematic review and meta-analysis. World J Clin Oncol 2017;8:273-84.

6. Hur H, Lee HY, Lee HJ, et al. Efficacy of laparoscopic subtotal gastrectomy with D2 lymphadenectomy for locally advanced gastric cancer: the protocol of the KLASS-02 multicenter randomized controlled clinical trial. BMC Cancer 2015;15:355.

7. Yu J, Huang C, Sun Y, et al. Effect of Laparoscopic vs Open Distal Gastrectomy on 3-Year Disease-Free Survival in Patients With Locally Advanced Gastric Cancer: The CLASS-01 Randomized Clinical Trial. JAMA 2019;321:1983-92.

8. Kanaya S, Gomi T, Momoi H, et al. Delta-shaped anastomosis in totally laparoscopic Billroth I gastrectomy: new technique of intraabdominal gastroduodenostomy. J Am Coll Surg 2002;195:284-7.

9. Wang SY, Hong J, Hao HK. A comparative study of deltashaped and conventional Billroth I anastomosis after laparoscopic distal gastrectomy for gastric cancer. Surg Endosc 2017;31:3191-202.

10. Lee HH, Song KY, Lee JS, et al. Delta-shaped anastomosis, a good substitute for conventional Billroth I technique with comparable long-term functional outcome in totally laparoscopic distal gastrectomy. Surg Endosc 2015;29:2545-52.

11. Japanese Gastric Cancer Association. Japanese gastric cancer treatment guidelines 2010 (ver. 3). Gastric Cancer 2011;14:113-23.

12. Nakajima T. Gastric cancer treatment guidelines in Japan. Gastric Cancer 2002;5:1-5.

13. Raziee HR, Cardoso R, Seevaratnam R, et al. Systematic review of the predictors of positive margins in gastric cancer surgery and the effect on survival. Gastric Cancer 2012;15 Suppl 1:S116-24.

14. Jemal A, Bray F, Center MM, et al. Global cancer statistics. CA Cancer J Clin 2011;61:69-90.

15. Ajani JA, Bentrem DJ, Besh S, et al. Gastric cancer, version 2.2013: featured updates to the NCCN Guidelines. J Natl Compr Canc Netw 2013;11:531-46.

16. Ajani JA, D'Amico TA, Almhanna K, et al. Gastric Cancer, Version 3.2016, NCCN Clinical Practice Guidelines in Oncology. J Natl Compr Canc Netw 2016;14:1286-312.

17. Clark CJ, Thirlby RC, Picozzi V Jr, et al. Current problems in surgery: gastric cancer. Curr Probl Surg 2006;43:566-670.

18. Van Cutsem E, Sagaert X, Topal B, et al. Gastric cancer. Lancet 2016;388:2654-64.

19. Japanese Gastric Cancer Association. Japanese gastric cancer treatment guidelines 2014 (ver. 4). Gastric Cancer 2017;20:1-19.

20. Deng K, Yang L, Hu B, et al. The prognostic significance of pretreatment serum CEA levels in gastric cancer: a meta-analysis including 14651 patients. PLoS One 2015;10:e0124151.

21. Qiao YF, Chen CG, Yue J, et al. Prognostic significance of preoperative and postoperative CK19 and CEA mRNA levels in peripheral blood of patients with gastric cardia cancer. World J Gastroenterol 2017;23:1424-33.

(English Language Editor: C. Betlazar-Maseh)
Cite this article as: Li J, Ge YG, Yang YF, Zhang J. Comparison of delta-shaped anastomosis and Billroth I reconstruction after laparoscopic distal gastrectomy for gastric cancer. J Gastrointest Oncol 2021;12(2):259-267. doi: 10.21037/ jgo-21-109 